\title{
Association of Mouse Mammary Tumor Virus With Human Breast Cancer: Histology, Immunohistochemistry and Polymerase Chain Reaction Analyses
}

OPEN ACCESS

Edited by:

Viive Maarika Howell,

University of Sydney, Australia

Reviewed by:

Carmine De Angelis, Baylor College of Medicine,

United States

Lianjin Jin,

Tulane University, United States Alexandra Allende,

Douglass Hanly Moir Pathology,

Australia

*Correspondence:

James S. Lawson

james.lawson@unsw.edu.au

these authors are co-senior authors.

Specialty section: This article was submitted to Women's Cancer,

a section of the journal

Frontiers in Oncology

Received: 30 January 2018 Accepted: 18 April 2018

Published: 07 May 2018

Citation: Lawson JS, Mazzanti C, Civita P, Menicagli M, Ngan CC, Whitaker NJ, Hochman J, Braitbard O, Yosufi B and Glenn WK (2018) Association of Mouse Mammary Tumor Virus With

Human Breast Cancer: Histology,

Immunohistochemistry and Polymerase Chain Reaction Analyses.

Front. Oncol. 8:141.

doi: 10.3389/fonc.2018.00141

\author{
James S. Lawson ${ }^{1 * t}$, Chiara Mazzanti ${ }^{2 \dagger}$, Prospero Civita ${ }^{2}$, Michele Menicagli², \\ Christopher C. Ngan', Noel J. Whitaker', Jacob Hochman', Ori Braitbard ${ }^{3}$, \\ Benafsha Yosufi' and Wendy K. Glenn' \\ 'University of New South Wales, Sydney, NSW, Australia, ${ }^{2}$ Fondazione Pisana per la Scienza Onlus, Pisa, Italy, \\ ${ }^{3}$ Department of Cell and Developmental Biology, Alexander Silberman Institute of Life Sciences, The Hebrew \\ University of Jerusalem, Jerusalem, Israel, ${ }^{4}$ Douglass Hanly Moir Pathology, Macquarie Park, NSW, Australia
}

Purpose: The purpose of this study is to determine whether mouse mammary tumor virus (MMTV)-associated human breast cancer has the same or similar histology to MMTV-associated mouse mammary tumors. Such associations may indicate a role for MMTV in human breast cancer.

Methods: Immunohistochemical techniques (using antibodies directed against the signal peptide p14 of the envelope precursor protein of MMTV) and polymerase chain reaction (PCR) analyses were used to identify MMTV proteins and MMTV-like envelope gene sequences in a series of breast cancers from Australian women. The histological characteristics of these human breast cancer specimens were compared with MMTV positive mouse mammary tumors. The same methods were used to study benign breast tissues which 1-11 years later developed into breast cancer.

Results: MMTV p14 proteins were identified in 27 (54\%) of 50 human breast cancers. MMTV env gene sequences were identified by PCR in 12 (27\%) of 45 human breast cancers. There was a significant correlation between the presence of MMTV (identified by $\mathrm{p} 14$ immunohistochemistry) in human breast cancers and histological characteristics similar to MMTV positive mouse mammary tumors $(p=0.001)$. There was a non-significant correlation between the presence of MMTV env gene sequences (identified by PCR) in human breast cancers and histological characteristics similar to MMTV positive mouse mammary tumors ( $p=0.290)$. MMTV p14 proteins were identified in $7(54 \%)$ of 13 benign breast specimens that later developed into human breast cancers. MMTV by PCR was identified in two benign specimens one of whom later developed MMTV positive breast cancer.

Discussion: These observations offer evidence that MMTV may be associated with characteristic human breast cancer histology. p14-based immunohistochemistry appears to be a more reliable technique than PCR for the identification of MMTV in human breast cancer. Identification of MMTV-associated p14 proteins in benign breast 
tissues confirms prior PCR-based studies that MMTV infection occurs before the development of MMTV positive breast cancer.

Conclusion: Many MMTV positive human breast cancers have similar histology to MMTV positive mouse mammary tumors. MMTV infection identified in benign breast tissues precedes development of MMTV positive human breast cancer. When considered in the context of prior studies, these observations indicate a likely role for MMTV in human breast cancer.

\begin{abstract}
Keywords: breast cancer, mouse mammary tumors, mouse mammary tumor virus, histology, mouse mammary tumor virus signal peptide, mouse mammary tumor virus p14, immunohistochemistry, polymerase chain reaction
\end{abstract}

\section{PURPOSE}

The purpose of this study is to determine whether mouse mammary tumor virus (MMTV)-associated human breast cancer has the same or similar histology to MMTV-associated mouse mammary tumors. Such associations may indicate a role for MMTV in human breast cancer. MMTVs have a documented causal role in mouse mammary tumors (1). Viral sequences, virtually identical to MMTV, have been repeatedly identified in human breast cancers by polymerase chain reaction (PCR) laboratory techniques and more recently by massive parallel sequencing $(2,3)$. The evidence suggestive of a role for an MMTV-like virus in human breast cancer is substantial but not conclusive.

\section{CONTEXT OF THIS STUDY}

It is helpful to place this current study in the context of past investigations. A “mouse milk factor," later identified as MMTV, was first associated with mouse mammary tumors by Bittner in 1936 (4). MMTV exerts oncogenic influences on both wild (feral) and inbred laboratory mice which leads to consistent histology patterns in mouse mammary tumors (5). During the 1950s, Thelma Dunn studied the histological characteristics of mammary tumors in wild and laboratory mice. Over $90 \%$ of mammary tumors in feral mice are adenocarcinomas. Dunn classified mouse mammary tumors as types A and B. Type B is by far the most common type. There are additional types of mouse mammary tumors including papillomas but these are uncommon.

The first observation that some human breast cancers had similar histology to MMTV-associated mouse mammary tumors was made by Wellings (6). However, others disagree that there are such similarities (7). The view that human breast cancer histology differs from mouse mammary tumors may be based on observations of advanced breast cancers. Many, but not all, advanced breast cancers are characterized by streams of elongated cancer cells surrounded by dense connective tissues. By way of contrast, the observations by Wellings appear to be based on the early proliferative stages of both human breast neoplasia and mouse mammary tumors (6). In our opinion, it is valid to compare these early stages of human breast cancer with mouse mammary tumors.

\section{COMPARISONS BETWEEN MMTV- ASSOCIATED MOUSE MAMMARY TUMORS AND MMTV-ASSOCIATED HUMAN BREAST CANCER}

There are close parallels between MMTV-associated cancer biology in both human breast cancer and mouse mammary tumors. (i) MMTV has been identified globally in human breast cancer and mouse mammary tumors (2, 8), (ii) MMTV virus particles have been identified in human breast cancers and mouse mammary tumors $(9,10)$, (iii) MMTV has been identified in both mouse and human milk $(1,11,12)$, (iv) nucleotide sequences and structure of the 9,900-bp long MMTV genome is $84-98 \%$ homologous in both mouse and human breast cancer $(3,13-16)$, (v) abnormal cancer related gene expression is similar $(17,18)$, (vi) MMTV-associated protein expression is similar (19-21), (vii) MMTV superantigen expression required for activating lymphocytes appears to be the same (22-24), (viii) MMTV infects intestinal B lymphocytes and randomly integrates into both the mouse and human genome (1, 25-27), (ix) hormone responsiveness appears to be similar (28-30), (x) MMTVassociated breast cancer histology may be similar $(5,6,31)$, (xi) there is a positive serological response to MMTV in both mouse and humans although this has not been confirmed by modern methods in humans (32-36), and (xii) MMTV is present in normal human breast and mouse mammary tissues prior to the development of MMTV positive breast cancer and mouse mammary tumors (37-39).

\section{MMTV p14 PROTEIN}

p14 is the signal peptide of the envelope precursor protein of MMTV (21). It has been developed by the Hochman group in Israel as an antibody which can be used for immunohistochemistry analyses to identify MMTV. In addition to being a signal peptide, it is a multifunctional 98 amino acid peptide. It binds and transcriptionally regulates key proteins of the cellular stress response and ribosome biogenesis. p14 can also function in both an oncogenic and an anti-oncogenic capacity depending on its phosphorylation status (40). Furthermore, p14 is expressed on the cell surface of murine mammary carcinomas and lymphomas that harbor MMTV (40). p14 has recently been located on the cell 
surface of primary human breast cancer cells grown in culture (41). The same human breast cancer cells were also positive for MMTV env sequences using PCR. p14 has been used for vaccination and passive immunization (using monoclonal antibodies and adoptive cell transfer) in MMTV positive mouse mammary tumors (41).

The use of MMTV p14 antibodies in immunohistochemistry analyses, offers improved consistency in the identification of MMTV in human breast cancer as compared to PCR techniques $(21,40,41)$.

\section{HYPOTHESIS}

We hypothesize that because of this almost identical cancerassociated biology between MMTV positive human breast cancer and mouse mammary tumors ${ }^{1}$, the cancer histology should also be similar. We have previously demonstrated that this hypothesis may be correct (31). However, in the previous study, correlations between human and mouse histology and the identification of MMTV, were not statistically significant (31). These insignificant results were probably due to the inconsistent identification of MMTV by PCR. Advances in identifying MMTV by immunohistochemistry using p14 antibody has added validity to the hypothesis.

\section{MATERIALS AND METHODS}

\section{Ethics}

This project was formally considered and approved by the Human Research Ethics Committee of the University of New South Wales (UNSW), Sydney, Australia. Ref: HC11421.

\section{Materials}

Fifty-five breast cancer specimens from Australian women were included in this study. All the specimens were from the archives of an Australian pathology service (Douglass Hanly MoirPathology) and had been formalin fixed and paraffin mounted. A study based on PCR analyses of MMTV in these specimens has previously been published (39). Additional specimens were invasive breast cancers from women who had previously had benign breast conditions and who several years later developed breast cancer. These specimens all differ from those used in our previous publications $(31,42)$.

Ten archival MMTV positive mouse mammary tumors in $\mathrm{C} 3 \mathrm{H}$ mice were from the Jackson laboratories (ME, USA).

\section{Investigations Based on PCR}

The DNA extraction and detection of MMTV-like env sequences were performed by PCR techniques as described by Wang et al. (43). These PCR analyses were conducted in three independent laboratories-the Icahn School of Medicine at Mount Sinai (ISMMS) (New York), the UNSW, Sydney, Australia, and the

${ }^{1}$ By informal convention, the term "mammary tumor," has been used to describe breast cancer in mice and other rodents.
Fondazione Pisana per la Scienza Onlus, Pisa, Italy. The results of these PCR analyses have previously been published (39). The primer sequences used in these PCR analyses include part of the MMTV env gene, which differs from human endogenous retrovirus 10 .

Contamination is a well-known problem with PCR analyses. Therefore, with respect to specimens analyzed at ISMMS, the reagents and PCR products were tested for the presence of murine mitochondrial (MoMt) and genomic DNA. The methods used were as described by Deligdisch et al. (44).

The same PCR methods were used to identify MMTVs in the 10 archival mouse mammary tumors. The results have previously been reported (45).

\section{Investigations Based on Immunohistochemistry}

p14 antibodies used in this current study were prepared as previously described using purified recombinant protein (21). This protein, designated p14 (based on Western blotting analyses), corresponds to the 98 amino acids of the MMTV envelope precursor signal peptide (46). Antibodies to p14 appear to be specific for the identification of MMTV $(21,40,41,46)$.

The immunohistochemical analyses were conducted on the same specimens in both the UNSW and the Fondazione Pisana per la Scienza Onlus, Pisa, Italy. The same methods were used in each independent laboratory. The immunohistochemical analyses were performed on $5 \mu \mathrm{m}$-thick paraffin sections. The antigen retrieval was achieved with MS-unmasker solution (DIAPATH, Martinengo, BG, Italy) in microwave. HistostainPlus kit (Invitrogen, Carlsbad, CA, USA) was used according to manufacturer's protocol. The slides were incubated for $2 \mathrm{~h}$ with a primary antibody, rabbit polyclonal anti-MMTV-p14 (1:500 dilution), then developed with diaminobenzidine chromogen (DAB) (DAKO, Glostrup, Denmark), and counterstained with hematoxylin. Negative controls included the omission of the primary antibody.

The results of immunohistochemical analyses for estrogen receptor (ER), progesterone receptor (PR), human epidermal growth factor receptor 2 (HER2), and p53 were available for each specimen as part of standard laboratory procedures at Douglass Hanly Moir Pathology - the source of the specimens. The grade of invasive breast cancers was available for 20 of the specimens.

\section{Immunohistochemistry Identification of Lymphocytes}

In human tissue specimens, lymphocytes can be readily identified by their histological characteristics, mainly because of their smaller diameter when compared to human breast cancer cells. In mouse tissue specimens, the diameter of mouse mammary tumor cells is approximately $20 \%$ less than human breast cancer cells. Therefore, when making comparisons of the histological characteristics between human and mouse mammary tumor cells, it is important to exclude lymphocytes. Lymphocytes plus normal and cancerous epithelial cells in human specimens can be identified by specific antibodies. In this study, CD45 antibodies (Dako M0701), which are specific for the identification of 
both T and B lymphocytes, were identified by automated immunochemistry techniques at the Douglass Hanly Moir-Pathology laboratories.

\section{Histological Comparisons Between Human Breast Cancer and Mouse Mammary Tumors}

The histological characteristics of both human breast cancer and mouse mammary tumor specimens were independently assessed by authors of this current study-James S. Lawson and Wendy K. Glenn. These specimens were sectioned and stained with hematoxylin and eosin (H\&E). The histological assessment was conducted "blind," that is, the histological assessments were conducted without knowledge of the outcomes of the PCR or immunohistochemistry p14 analyses. When viewed macroscopically human breast cancer and mouse mammary tumors have different characteristics. When examined microscopically at a magnification of 100,200, and 400, some human breast cancers and mouse mammary tumor tissues are very similar (31).

Comparisons were also made between the histology of 12 human benign breast specimens which 1-11 years later developed into breast cancer.

\section{Statistics}

The Pearson's correlation test was used to determine if there were correlations between the identification of MMTV by immunohistochemistry (using p14 antibodies) and PCR and (i) specific histological features in human breast cancer and (ii) biomarkers including ERs, PRs, HER2, and p53. A p-value of less than 0.05 was considered to be significant. The SPSS statistical package was used.

\section{RESULTS}

\section{Comparisons of Histology Between MMTV Positive Human Breast Cancer and MMTV Positive Mouse Mammary Tumors}

Mouse mammary tumor virus p14 proteins were identified in 27 (54\%) of 50 human breast cancers. MMTV env gene sequences were identified by PCR in 12 (27\%) of 45 human breast cancers. There was a significant correlation between the presence of MMTV (determined by immunohistochemistry using p14 antibodies) in human breast cancers and histological characteristics similar to MMTV positive mouse mammary tumors $(p=0.033)$ based on UNSW laboratory data, $p=0.001$ using the combined data from Pisa and UNSW (Table S1 in Supplementary Material).

There was no correlation between the presence of MMTV env gene sequences identified by PCR in human breast cancers and histological characteristics similar to MMTV positive mouse mammary tumors $(p=0.290)$. There was no correlation between the identification of MMTV by immunohistochemistry using p14 antibodies and PCR $(p=0.932)$.

These results are shown in Tables $\mathbf{1}$ and $\mathbf{2}$ and Tables S1-S3 in Supplementary Material. There are gaps in the data shown in Table S2 in Supplementary Material, these are due to the absence
TABLE 1 | Human breast cancer.

\begin{tabular}{lcc}
\hline & $\begin{array}{c}\text { Histology Dunn } \\
\text { positive }\end{array}$ & $\begin{array}{c}\text { Histology Dunn } \\
\text { negative }\end{array}$ \\
\hline MMTV by p14 positive & 19 & 8 \\
MMTV by p14 negative & 6 & 17
\end{tabular}

Correlation between identification of MMTV by p14 immunohistochemistry (Pisa and University of New South Wales data combined) and histological characteristics. Breast cancer histology assessed as similar or not similar to MMTV positive mouse mammary tumors Dunn types $A$ and $B$. There is a significant correlation between the presence or absence of MMTV by immunohistochemistry using p14 antibodies and histological characteristics ( $p=0.001$ significant).

MMTV, mouse mammary tumor virus.

TABLE 2 | Human breast cancer.

\begin{tabular}{lcc}
\hline & $\begin{array}{c}\text { Histology Dunn } \\
\text { positive }\end{array}$ & $\begin{array}{c}\text { Histology Dunn } \\
\text { negative }\end{array}$ \\
\hline MMTV by PCR positive & 8 & 4 \\
MMTV by PCR negative & 16 & 17 \\
\hline
\end{tabular}

Correlation between identification of MMTV by PCR and histological characteristics. Breast cancer histology assessed as similar or not similar to MMTV positive mouse mammary tumors Dunn types $A$ and $B$. There is no correlation between the presence or absence of MMTV identified by PCR and histological characteristics ( $p=0.290$ not significant).

MMTV, mouse mammary tumor virus; PCR, polymerase chain reaction.

of beta-globin or exhaustion of the specimen blocks. There were no differences between invasive and non-invasive breast neoplasia with respect to their similarity or non-similarity with mouse mammary tumor histology (Table S2 in Supplementary Material).

These observations offer evidence that MMTV may be associated with characteristic human breast cancer histology.

Dunn type A MMTV positive human breast cancers have intensely stained nuclei with intact alveolar milk acini (31). Dunn type B MMTV positive human breast cancers are also characterized by intensely stained nuclei which occupy most of the cell (31). These cells are round and regular in size and are clumped together without glandular acini or lumina (31).

A typical MMTV positive human breast cancer specimen with Dunn type B histological characteristics compared to MMTV positive Dunn type B mouse mammary tumor is shown in Figures 1 and 2. A series of human breast cancers with histological characteristics similar to mouse Dunn type B are shown in Figure 3. The numbers on each image corresponds to patients listed in Table S2 in Supplementary Material. Typical Dunn type B human breast cancers are much more common than Dunn type A.

The identification of MMTV by immunohistochemistry using p14 antibodies in both human breast cancer and mouse mammary tumors is shown in Figure 4. There is staining in the cell membranes, cytoplasm, and in some but not all nuclei.

\section{Infiltrating Lymphocytes}

Infiltrating lymphocytes were identified in many, but not all, human breast cancer specimens. These lymphocytes could be clearly identified by their histological characteristics and red staining-based immunohistochemistry using antibodies to B and T cells (Figure 5). 

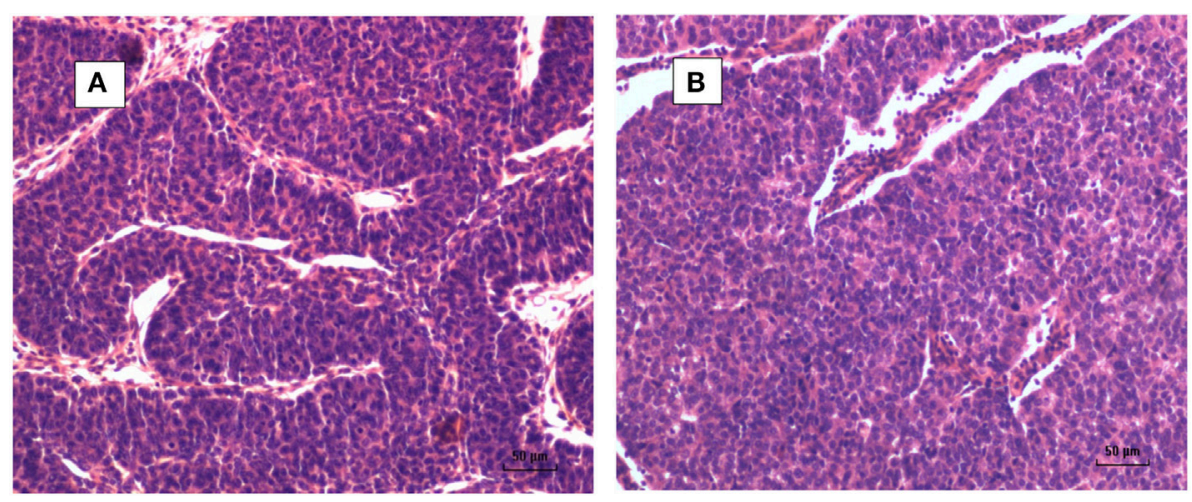

FIGURE 1 | (A) Mouse mammary tumor-Dunn type B, mouse mammary tumor virus (MMTV) positive by polymerase chain reaction (PCR). (B) Human invasive ductal carcinoma - similar histology to Dunn type B mouse mammary tumor. MMTV positive by PCR.
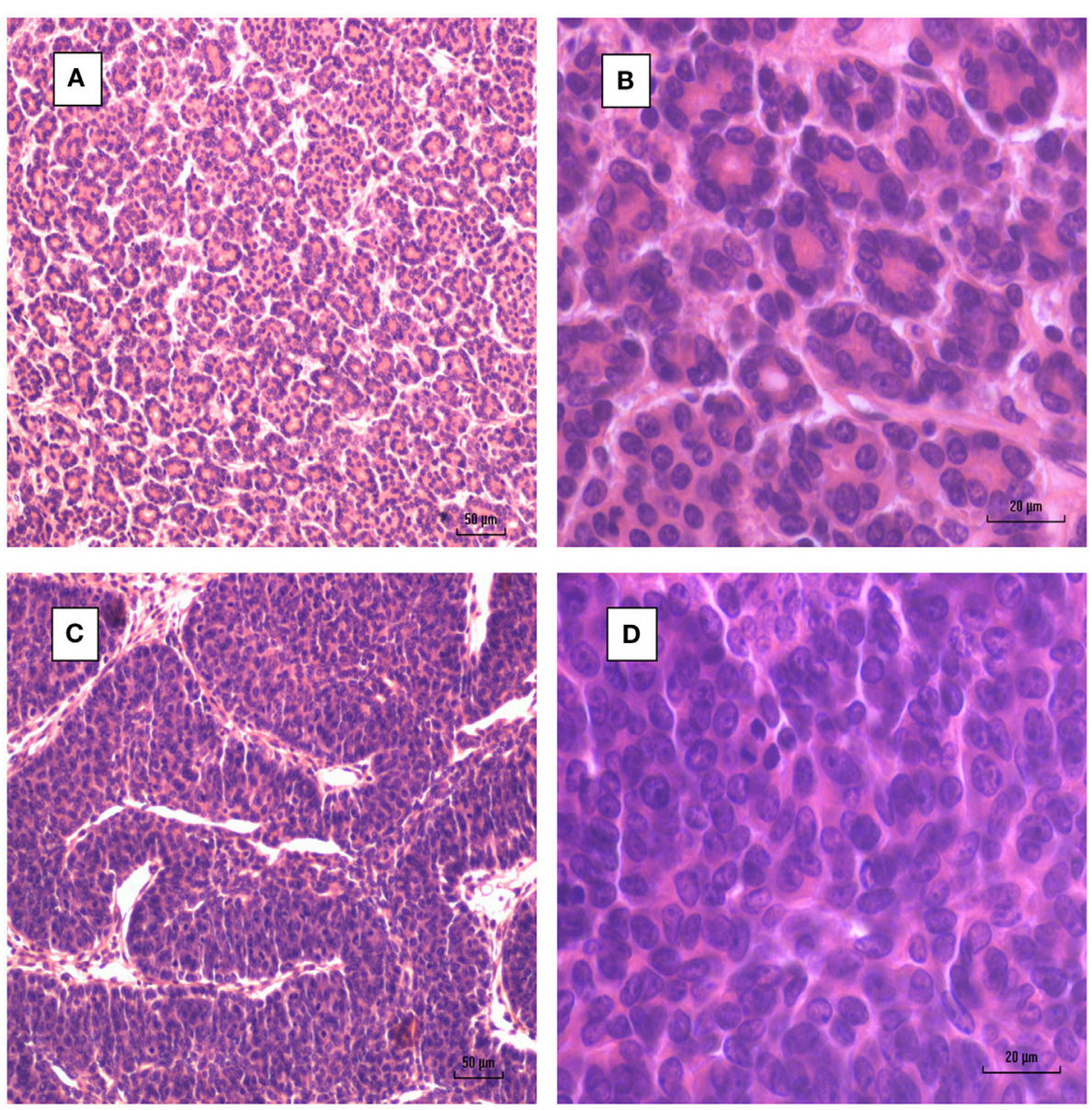

FIGURE 2 | Mouse mammary tumors. (A,B) Dunn type A. (C,D) Dunn type B. Dunn type B is much more prevalent than Dunn type A.

\section{Comparisons Between Human Benign Breast Specimens Which 1-11 Years Later Developed Into Breast Cancer \\ There were 13 sets of benign breast specimens which $1-11$ years} later developed into breast cancer. 11 of these 13 benign breast specimens had some hyperplasia (as defined by four or more levels of epithelial cells). MMTV-p14 was identified in 8 of the 13 benign breast specimens which later developed into breast cancer in the same women. Six of the later breast cancers were MMTV-p14 positive. MMTV-p14 was identified in 8 of the 11 benign breast specimens with hyperplasia, 7 of which 

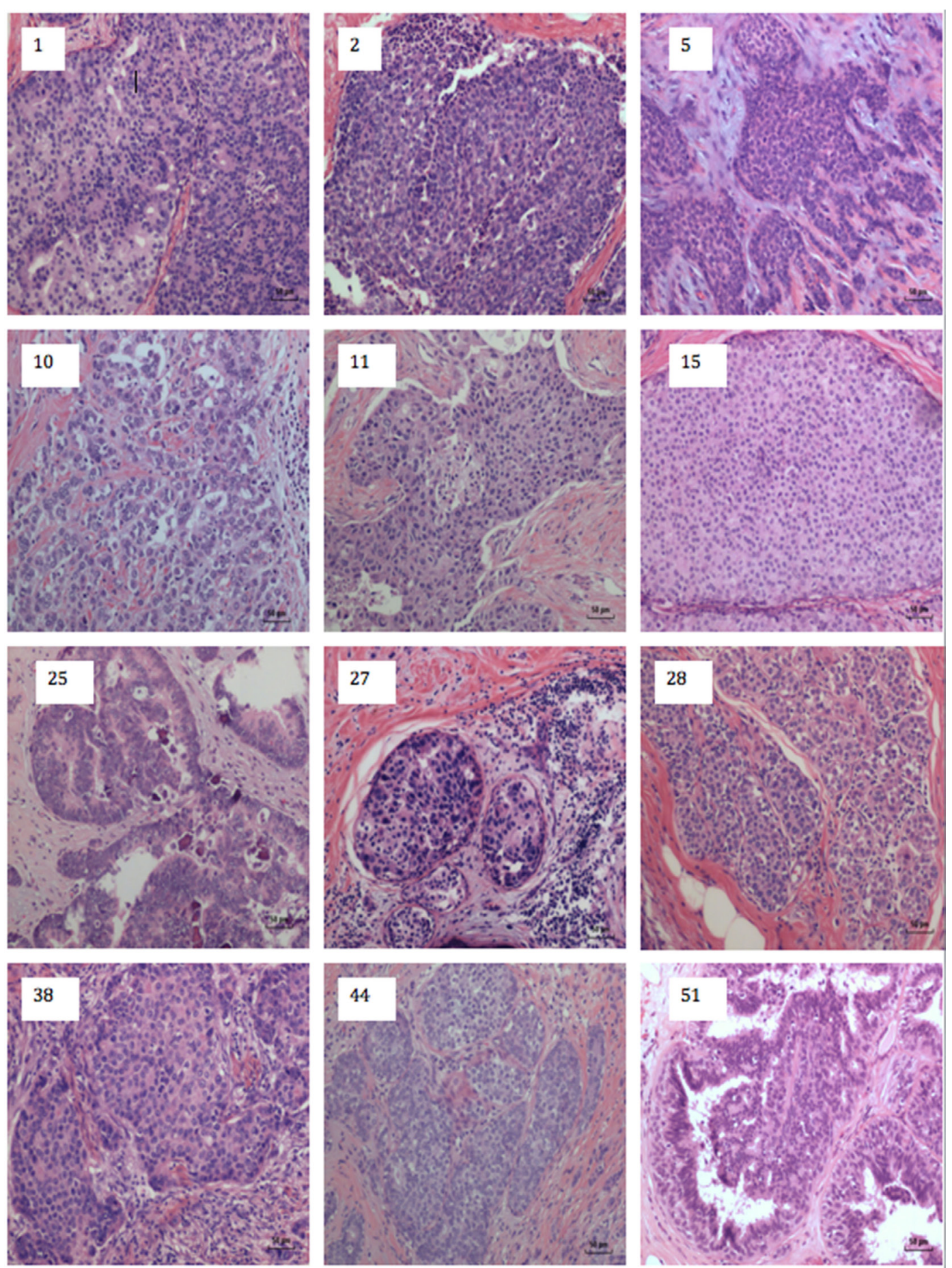

FIGURE 3 | Human breast cancer specimens with histological characteristics similar to mouse mammary tumors. The numbers on each image refers to the patient numbers listed in Table $\mathbf{S} 1$ in Supplementary Material.

subsequently developed MMTV-p14 positive breast cancer. The details are shown in Table S3 in Supplementary Material. An MMTV positive benign breast specimen with hyperplasia progressing to MMTV positive breast invasive ductal carcinoma is shown in Figure 6.

\section{Consistency and Specificity of Immunohistochemistry p14 Analyses Between Laboratories}

As shown in Table S2 in Supplementary Material, the identification of MMTV by immunohistochemistry using p14 antibodies 

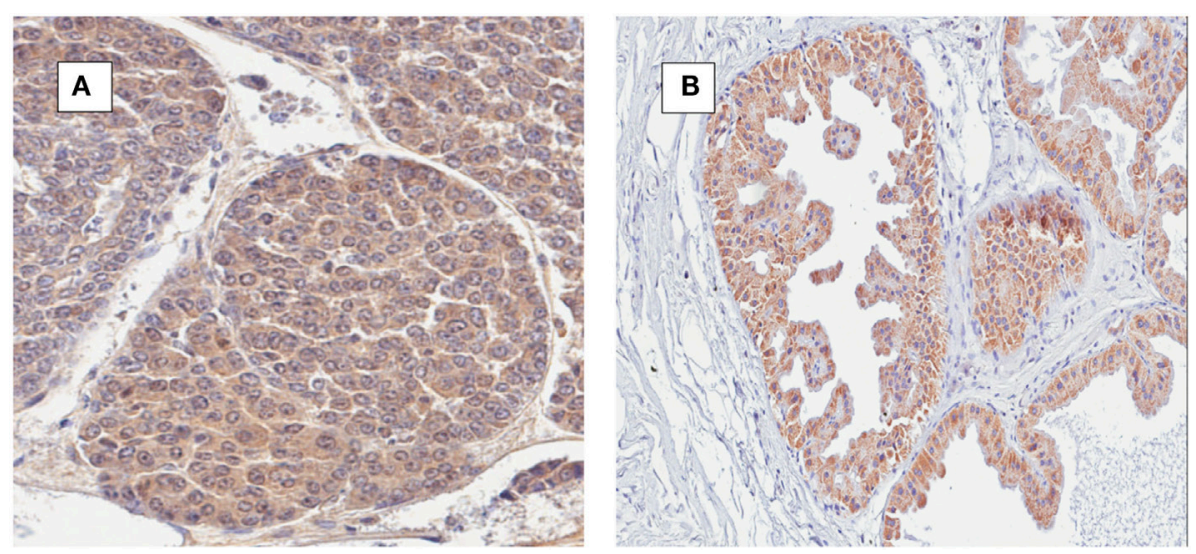

FIGURE 4 | (A) Mouse mammary tumor; mouse mammary tumor virus (MMTV) p14 protein positive. (B) Human ductal carcinoma in situ; MMTV p14 protein positive.

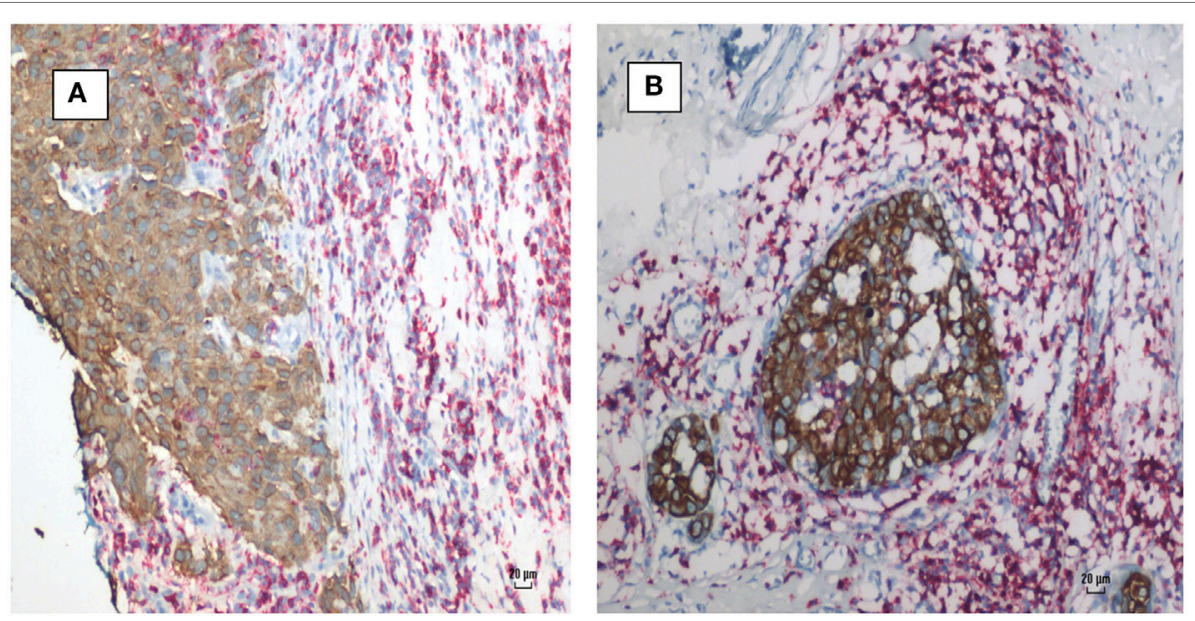

FIGURE 5 | Mouse mammary tumor virus positive human breast cancer. Breast cancer cells (brown stain) and lymphocytes (red stain) by immunohistochemistry using CD45 antibodies. (A) Invasive ductal carcinoma. (B) Ductal carcinoma in situ. Lymphocytes surround and infiltrate both cancers.
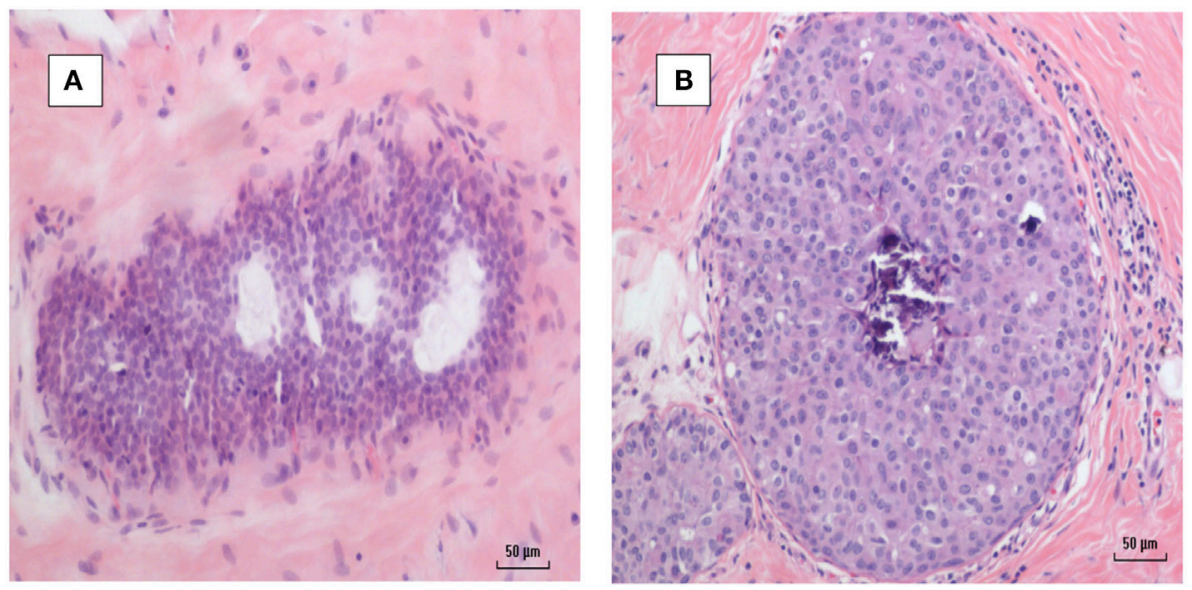

FIGURE 6 | (A) Mouse mammary tumor virus (MMTV) positive by polymerase chain reaction (PCR) breast hyperplasia (B) MMTV positive by PCR subsequent ductal carcinoma in situ breast cancer-same patient. 
is higher than identification by PCR. The identification of MMTV by immunohistochemistry in the Pisa laboratory as compared to the Sydney laboratory (same specimens, same p14 antibody) was the same for 29 specimens and different for 6 specimens.

As shown in Figure 7, identification of MMTV by immunohistochemistry using p14 antibodies in patients 5 and 6 listed in Table S2 in Supplementary Material indicates specificity of the p14 antibody. If cross reactivity of the p14 was occurring, the cancer specimen from patient 6 should not be negative.

\section{Correlations Between MMTV Identification in Human Breast Cancer, Cancer Grade, and Biomarkers}

The results of immunohistochemical analyses for ER, PR, HER2, and p53 are shown in Table S1 in Supplementary Material. There were no significant correlations with respect these biomarkers and MMTV identification in breast cancer by either immunohistochemistry or PCR.

There were no correlations between the breast cancer grade and MMTV identification by either p14 immunohistochemistry or PCR ( $p=0.586$ and 0.719 , respectively).

\section{DISCUSSION}

Mouse mammary tumor virus $\mathrm{p} 14$ proteins were identified in 25 (50\%) of 50 human breast cancers. MMTV env gene sequences were identified by PCR in $12(27 \%)$ of 45 of the same human breast cancers. There was a significant correlation between the presence of MMTV (determined by immunohistochemistry using p14 antibodies) in human breast cancers and histological characteristics similar to MMTV positive mouse mammary tumors. This correlation was confirmed in two laboratories ( $p=0.033$ UNSW, $p=0.001$ Pisa). There was no correlation between the presence of MMTV env gene sequences (determined by PCR) in human breast cancers and histological characteristics similar to MMTV positive mouse mammary tumors $(p=0.290)$. There was no correlation between PCR and p14 used for the identification of MMTV $(p=0.932)$.

Identification of MMTV-associated p14 proteins in benign breast tissues confirms prior PCR-based studies that MMTV infection occurs before the development of MMTV positive breast cancer.

These observations offer evidence (i) that MMTV may be associated with characteristic human breast cancer histology and
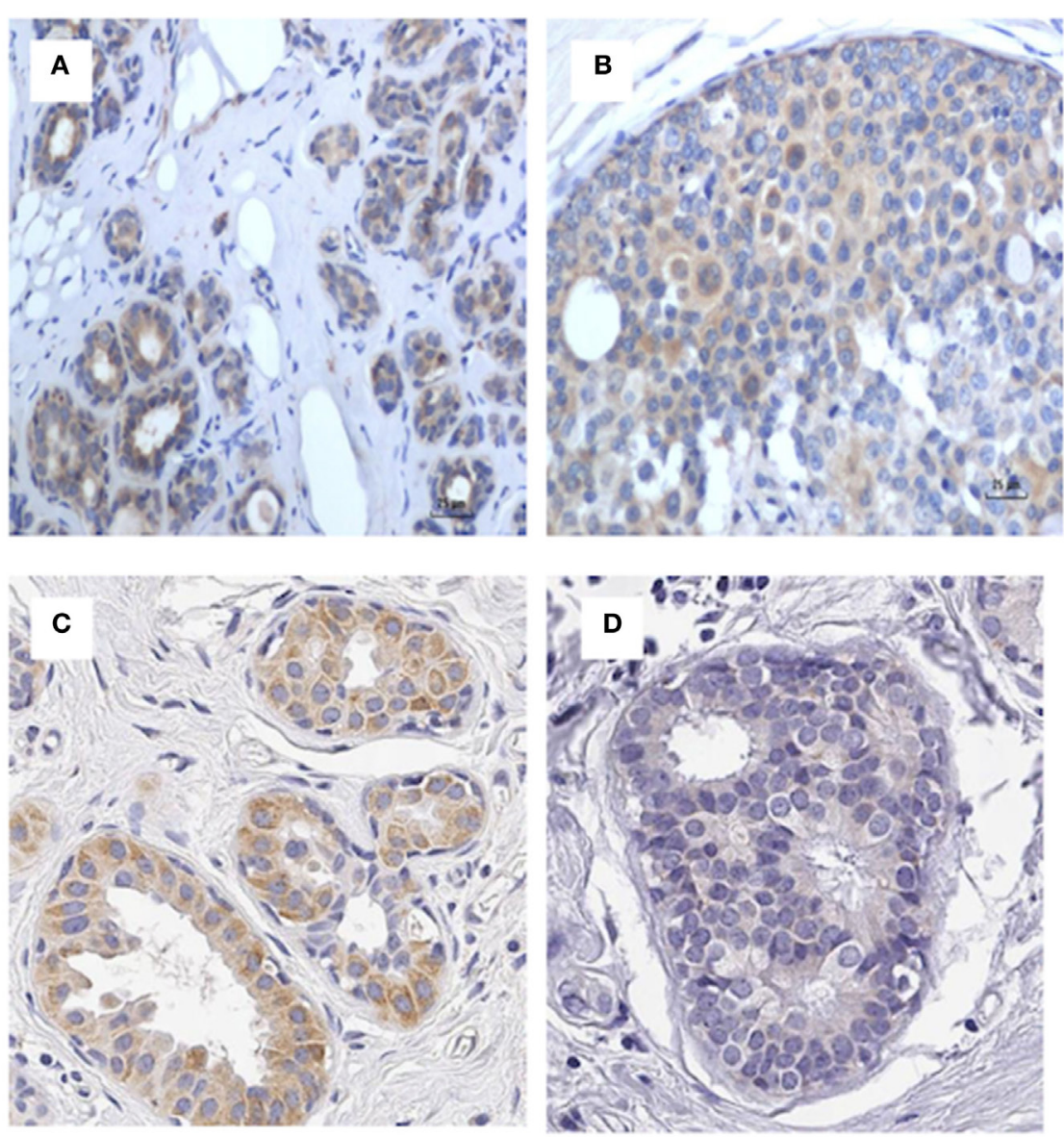

FIGURE 7 | p14 identification in patients 5 and 6 in Table S3 in Supplementary Material indicating specificity of the P14 antibody. (A) Patient 5 benign (positive), (B) patient 5 cancer (positive), (C) patient 6 benign (positive), (D) patient 6 cancer (negative). If cross reactivity of the p14 was occurring, the cancer specimen from patient 6 should not be negative. 
(ii) that MMTV infection precedes the development of MMTVassociated human breast cancer.

There are qualifications with respect to this evidence: (i) due to extremely low levels of viral DNA, the identification of MMTV by PCR in human breast cancers is not consistent, (ii) the assessment of histological characteristics is subjective, and (iii) the assessment of staining used in immunohistochemistry is also subjective (but no less subjective than other analyses based on immunohistochemistry, such as ER and PR protein expression in breast cancer).

\section{Validity of the Observations}

As the p14-based assessments were conducted in two independent laboratories with similar outcomes, the results have added validity.

The identification of extremely low concentrations of retroviral DNA such as MMTV in human breast cancer by PCR is difficult and the results are inconsistent. The results of PCR analyses for the identification of MMTV using the same breast cancer specimens can differ between laboratories (39). There are several reasons for the inconsistent outcomes when PCR is used for the identification of MMTV in human breast cancers: (i) the MMTV retroviral genome consists of approximately 10,000 bp which is very small as compared to the host human genome of 3 billion $(3,000,000,000) \mathrm{bp}$, (ii) the infected cancer cells may be only a small fraction of the cancer specimens, and (iii) the infected cells may contain a low number of the MMTV genome (47). These difficulties can lead to false negative outcomes and probably accounts for the low identification of MMTV by PCR in this current study.

Despite the inconsistent outcomes, results based on PCR are valid for the following reasons: (i) there were variations in approximately $3 \%$ of the MMTV env gene sequences which is an indication that contamination is unlikely, (ii) the primers used in PCR analyses are based on MMTV gene sequences which are unique to the MMTV genome, (iii) there was no contamination by mouse gene sequences as demonstrated by the absence of MoMt or IAP DNA sequences [these analyses were conducted in the Mount Sinai Medical Center, New York and have been previously published (39)]. In addition, the results based on PCR are consistent with prior studies of MMTV in Australian breast cancers $(42,48)$. These results are also consistent with US and Italian-based studies $(43,49)$.

While the antibodies to p14 appear to be specific for the identification of MMTV, it cannot be excluded that some false positive outcomes could occur due to cross-reacting proteins. However, as shown in Figure 7 there was both positive and negative identification of MMTV in benign and breast cancer specimens by immunohistochemistry using p14 antibody, which indicates that p14 antibody is likely to be specific The similar results between the two independent laboratories (Pisa and the University of NSW) is a strong indication that p14-based immunohistochemistry is a more reliable technique than PCR for the identification of MMTV in human breast cancer.

The comparisons of the histologic characteristics of human breast cancers and mouse mammary tumors have obvious subjective elements. Importantly, other cancers such as basal cell carcinomas of the skin have similar characteristics to MMTV positive human breast cancer. For this reason the diagnosis of MMTV involvement in human breast cancer cannot be made on histological characteristics in isolation from additional laboratory evidence. However, the two assessors (James S. Lawson and Wendy K. Glenn) could not distinguish between the MMTV positive human breast cancers and mouse mammary tumors at a microscopic level. At a macroscopic level, human and mouse breast cancers differ.

There are precedents for viral infections leading to cancers with specific histological characteristics. The best known are (i) the association between human papilloma virus infections and koilocytes (cells with characteristic haloes surrounding the nucleus) and (ii) Reed-Sternberg cells (enlarged lymphocytes sometimes with multiple nuclei) in Hodgkin lymphomas. With respect to MMTV and histological characteristics, the similarity between MMTV positive human breast cancers and MMTV positive mouse mammary tumors may hint toward a similar tumor-microenvironment interaction.

The histological characteristics of MMTV-associated human breast cancer are similar to those of neuro-endocrine breast cancers. However, it has been demonstrated that MMTV is not associated with these specific breast cancers (50).

Cancer cells were clearly identified from lymphocytes by their different diameter and appearance and by immunohistochemistry. This confirms that the comparisons between MMTV positive human and mouse mammary tumors are not confused by the presence of lymphocytes.

The observation that MMTV p14 protein was identified by immunohistochemistry in $8(62 \%)$ of the 13 prior benign human breast specimens and that each had some characteristics of hyperplasia and that 7 developed MMTV positive Dunn type B human breast cancers is of considerable interest. This confirms the prior observations (on the same specimens) based on PCR (39). This suggests an initial infection by MMTV in benign breast tissues prior to the development of breast cancer in the same individual patients. In addition, the presence of hyperplasia associated with MMTV infection in benign breast tissues suggests an increase in risk of breast cancer. This is the first association of MMTV with this phenomenon.

Based on the present and previous reports $(21,41)$, we propose p14 as a potential diagnostic tool, as well as a putative predictor of MMTV-associated breast cancer development. Further studies with larger cohorts of patients in different laboratories will be needed to validate our findings. Taken together with our previous studies on murine MMTV-associated tumors (21, 40, $41,46)$, we also propose the use of p14-mediated strategies for preventive and therapeutic implications toward MMTVassociated breast cancers. This may be of special significance in the case of MMTV benign hyperplasia where preventive vaccination might be considered in clinical settings.

\section{Correlations Between MMTV Identification in Human Breast Cancer, Cancer Grade, and Biomarkers}

The absence of any correlations between MMTV positive breast cancer and the biomarkers ER, PR, HER2, and p53 confirms the 
prior observations by the Pogo group (51). The absence of any correlations between the breast cancer stage and MMTV identification also confirms the prior observations (51).

\section{CONCLUSION}

We hypothesized that because of the close parallels in the biology of MMTV-associated human breast cancer and mouse mammary tumors, there should be consistent histological patterns in MMTV positive human breast cancers which are similar to MMTV-associated mouse mammary tumors. We have shown that this hypothesis is probably true. We have also confirmed by immunohistochemistry analysis using p14 antibodies that MMTV infections of benign breast tissues occurs before the development of MMTV positive breast cancer. When considered in the context of prior published evidence, the implication of these observations is that MMTV is likely to have a role in human breast cancer.

\section{ETHICS STATEMENT}

This project was formally considered and approved by the Human Research Ethics Committee of the University of New South Wales, Sydney, Australia. Reference: HC11421.

\section{REFERENCES}

1. Dudley JP, Golovkina TV, Ross SR. Lessons learned from mouse mammary tumor virus in animal models. ILARJ (2016) 57:12-23. doi:10.1093/ilar/ilv044

2. Wang F, Hou J, Shen Q, Yue Y, Xie F, Wang X, et al. Mouse mammary tumor virus-like virus infection and the risk of human breast cancer: a meta-analysis. Am J Transl Res (2014) 6:248-66.

3. Tang KW, Alaei-Mahabadi B, Samuelsson T, Lindh M, Larsson E. The landscape of viral expression and host gene fusion and adaptation in human cancer. Nat Commun (2013) 4:2513. doi:10.1038/ncomms3513

4. Bittner JJ. Some possible effects of nursing on the mammary gland tumor incidence in mice. Science (1936) 84:162. doi:10.1126/science.84.2172.162

5. Dunn T. Morphology of mammary tumors in mice. In: Homburger F, editor. Physiopathology of Cancer. New York: A. J. Phiebig (1959). p. 38-83.

6. Wellings SR. A hypothesis of the origin of human breast cancer from the terminal ductal lobular unit. Pathol Res Pract (1980) 166:515-35. doi:10.1016/ S0344-0338(80)80248-2

7. Cardiff RD, Munn RJ. Comparative pathology of mammary tumorigenesis in transgenic mice. Cancer Lett (1995) 90:13-9. doi:10.1016/0304-3835 (94)03672-6

8. Stewart THM, Sage RD, Stewart AFR, Cameron DW. Breast cancer incidence highest in the range of one species of house mouse, Mus domesticus. Br J Cancer (2000) 82:446-51. doi:10.1054/bjoc.1999.0941

9. Moore DH, Charney J, Kramarsky B, Lasfargues EY, Sarkar NH, Brennan MJ, et al. Search for a human breast cancer virus. Nature (1971) 229:611-4. doi:10.1038/229611a0

10. Feller WF. Human breast milk in relationship to breast cancer. CA Cancer J Clin (1968) 18:336-40. doi:10.3322/canjclin.18.6.336

11. Johal H, Ford CE, Glenn WK, Heads J, Lawson JS, Rawlinson WD. Mouse mammary tumor like virus (MMTV) sequences in breast milk from healthy lactating women. Breast Cancer Res Treat (2011) 129:149-55. doi:10.1007/ s10549-011-1421-6

12. Nartey T, Moran H, Marin T, Arcaro KF, Anderton DL, Etkind P, et al. Human mammary tumor virus (HMTV) sequences in human milk. Infect Agent Cancer (2014) 9:20. doi:10.1186/1750-9378-9-20

13. Moore R, Dixon M, Smith R, Peters G, Dickson C. Complete nucleotide sequence of a milk-transmitted mouse mammary tumor virus: two frameshift

\section{AUTHOR CONTRIBUTIONS}

$\mathrm{JL}, \mathrm{JH}$, and $\mathrm{CM}$ conceived the study. BY prepared the specimens for analysis. PC, MM, and CM conducted the p14-based immunohistochemistry analyses in Pisa, Italy. CN and WG conducted the p14-based immunohistochemistry analyses in Sydney, NSW, Australia. NW supervised the quality control of analyses and specimen assessments in Sydney. JH and OB developed the p14 antibody, conducted quality controls. JL, CN, and WG conducted the histological assessments. All authors were involved in the writing and final approval of the manuscript.

\section{ACKNOWLEDGMENTS}

The archival specimens were provided by the Douglass Hanly Moir Pathology Laboratories of Sydney, NSW, Australia. Additional immunohistochemistry analyses were conducted by Dinh D. Tran.

\section{SUPPLEMENTARY MATERIAL}

The Supplementary Material for this article can be found online at https://www.frontiersin.org/articles/10.3389/fonc.2018.00141/ full\#supplementary-material.

suppression events are required for translation of gag and pol. J Virol (1987) 61:480-90.

14. Nishio M, Xu L, Sasaki M, Haga S, Okumoto M, Mori N, et al. Complete nucleotide sequence of mouse mammary tumor virus from JYG Chinese wild mice: absence of bacterial insertion sequences in the cloned viral gag gene. Breast Cancer (1994) 1:89-94. doi:10.1007/BF02967037

15. Liu B, Wang Y, Melana SM, Pelisson I, Najfeld V, Holland JF, et al. Identification of a proviral structure in human breast cancer. Cancer Res (2001) 61:1754-9.

16. Melana SM, Nepomnaschy I, Sakalian M, Abbott A, Hasa J, Holland JF, et al. Characterization of viral particles isolated from primary cultures of human breast cancer cells. Cancer Res (2007) 67:8960-5. doi:10.1158/0008-5472. CAN-06-3892

17. Callahan R, Mudunur U, Bargo S, Raafat A, McCurdy D, Boulanger C, et al. Genes affected by mouse mammary tumor virus (MMTV) proviral insertions in mouse mammary tumors are deregulated or mutated in primary human mammary tumors. Oncotarget (2012) 3:1320-34. doi:10.18632/ oncotarget.682

18. Klein A, Wessel R, Graessmann M, Jürgens M, Petersen I, Schmutzler R, et al. Comparison of gene expression data from human and mouse breast cancers: identification of a conserved breast tumor gene set. Int J Cancer (2007) 121:683-8. doi:10.1002/ijc.22630

19. Keydar I, Mesa-Tejada R, Ramanarayanan M, Ohno T, Fenoglio C, Hu R, et al. Detection of viral proteins in mouse mammary tumors by immunoperoxidase staining of paraffin sections. Proc Natl Acad Sci U S A (1978) 75: 1524-8. doi:10.1073/pnas.75.3.1524

20. Melana SM, Nepomnaschy I, Hasa J, Djougarian A, Djougarian A, Holland JF, et al. Detection of human mammary tumor virus proteins in human breast cancer cells. J Virol Methods (2010) 163:157-61. doi:10.1016/j.jviromet.2009. 09.015

21. Bar-Sinai A, Bassa N, Fischette M, Gottesman MM, Love DC, Hanover JA, et al. Mouse mammary tumor virus Env-derived peptide associates with nucleolar targets in lymphoma, mammary carcinoma, and human breast cancer. Cancer Res (2005) 65:7223-30. doi:10.1158/0008-5472. CAN-04-3879

22. Labrecque N, McGrath H, Subramanyam M, Huber BT, Sékaly RP. Human $\mathrm{T}$ cells respond to mouse mammary tumor virus-encoded superantigen: 
V beta restriction and conserved evolutionary features. J Exp Med (1993) 177:1735-43. doi:10.1084/jem.177.6.1735

23. Wei WZ, Gill RF, Wang H. Mouse mammary tumor virus associated antigens and superantigens - immuno-molecular correlates of neoplastic progression. Semin Cancer Biol (1993) 4:205-13.

24. Wang Y, Jiang JD, Xu D, Li Y, Qu C, Holland JF, et al. A mouse mammary tumor virus-like long terminal repeat superantigen in human breast cancer. Cancer Res (2004) 64:4105-11. doi:10.1158/0008-5472.CAN-03-3880

25. Lushnikova AA, Kryukova IN, Rotin DL, Lubchenko LN. Detection of the env MMTV-homologous sequences in mammary carcinoma patient intestine lymphoid tissue. Dokl Biol Sci (2004) 399:423-6. doi:10.1007/s10630005-0001-5

26. Indik S, Günzburg WH, Salmons B, Rouault F. Mouse mammary tumor virus infects human cells. Cancer Res (2005) 65:6651-9. doi:10.1158/00085472.CAN-04-2609

27. Faschinger A, Rouault F, Sollner J, Lukas A, Salmons B, Günzburg WH. Mouse mammary tumor virus integration site selection in human and mouse genomes. J Virol (2008) 82:13. doi:10.1128/JVI.02098-07

28. Svec J, Hlavay E, Matoska J, Thurzo V. Hormone-responsive genes of the mouse mammary tumor virus. Czech Med (1979) 2:198-212.

29. Briand P. Hormone-dependent mammary tumors in mice and rats as a model for human breast cancer. Anticancer Res (1983) 3:273-81.

30. Wang Y, Melana SM, Baker B, Bleiweiss I, Fernandez-Cobo M, Mandeli JF, et al. High prevalence of MMTV-like env gene sequences in gestational breast cancer. Med Oncol (2003) 20:233-6. doi:10.1385/MO:20:3:233

31. Lawson JS, Tran DD, Carpenter E, Ford CE, Rawlinson WD, Whitaker NJ, et al. Presence of mouse mammary tumour-like virus gene sequences may be associated with specific human breast cancer morphology. J Clin Pathol (2006) 59:1287-92. doi:10.1136/jcp.2005.035907

32. Arthur LO, Bauer RF, Orme LS, Fine DL. Coexistence of the mouse mammary tumor virus (MMTV) major glycoprotein and natural antibodies to MMTV in sera of mammary tumor-bearing mice. Virology (1978) 87:266-75. doi:10.1016/0042-6822(78)90132-0

33. Day NK, Witkin SS, Sarkar NH, Kinne D, Jussawalla DJ, Levin A, et al. Antibodies reactive with murine mammary tumor virus in sera of patients with breast cancer: geographic and family studies. Proc Natl Acad Sci U S A (1981) 78:2483-7. doi:10.1073/pnas.78.4.2483

34. Lopez-Cepero M, Wang Y, Keydar I, Brandt-Carlson C, Butel JS, Lopez DM. Detection of retroviral superantigen and products of the envelope gene from endogenous mouse mammary tumor virus in $B$ cells from $B A L B / c$ mice. Cell Immunol (1995) 163:191-7. doi:10.1006/cimm.1995.1116

35. Dion AS, Girardi AJ, Williams CC, Pomenti AA. Serologic responses to murine mammary tumor virus (MuMTV) in MuMTV-exposed laboratory personnel. J Natl Cancer Inst (1986) 76:611-9. doi:10.1093/jnci/76.4.611

36. Goedert JJ, Rabkin CS, Ross SR. Prevalence of serologic reactivity against four strains of mouse mammary tumour virus among US women with breast cancer. Br J Cancer (2006) 94:548-51. doi:10.1038/sj.bjc.6602977

37. Rongey RW, Hlavackova A, Lara S, Estes J, Gardner MB. Types B and C RNA virus in breast tissue and milk of wild mice. J Natl Cancer Inst (1973) 50:1581-9. doi:10.1093/jnci/50.6.1581

38. Imai S. Mouse mammary tumor virus and mammary tumorigenesis in wild mice. Pathol Int (1996) 46:919-32. doi:10.1111/j.1440-1827.1996.tb03570.x

39. Nartey T, Mazzanti CM, Melana S, Glenn WK, Bevilacqua G, Holland JF, et al. Mouse mammary tumor-like virus (MMTV) is present in human breast tissue before development of virally associated breast cancer. Infect Agent Cancer (2017) 12:1. doi:10.1186/s13027-016-0113-6
40. Feldman D, Roniger M, Bar-Sinai A, Braitbard O, Natan C, Love DC, et al. The signal peptide of mouse mammary tumor virus-env: a phosphoprotein tumor modulator. Mol Cancer Res (2012) 10:1077-86. doi:10.1158/1541-7786. MCR-11-0581

41. Braitbard O, Roniger M, Bar-Sinai A, Rajchman D, Gross T, Abramovitch H, et al. A new immunization and treatment strategy for mouse mammary tumor virus (MMTV) associated cancers. Oncotarget (2016) 7:21168-80. doi:10.18632/oncotarget.7762

42. Lawson JS, Glenn WK, Salmons B, Ye Y, Heng B, Moody P, et al. Mouse mammary tumor virus-like sequences in human breast cancer. Cancer Res (2010) 70:3576-85. doi:10.1158/0008-5472.CAN-09-4160

43. Wang Y, Jiang JD, Xu D, Li Y, Qu C, Holland JF, et al. Detection of mammary tumor virus env gene-like sequences in human breast cancer. Cancer Res (1995) 55:5173-9.

44. Deligdisch L, Marin T, Lee AT, Etkind P, Holland JF, Melana S, et al. Human mammary tumor virus (HMTV) in endometrial carcinoma. Int J Gynecol Cancer (2013) 23:1423-8. doi:10.1097/IGC.0b013e3182980fc5

45. Glenn WK, Lawson JS, Whitaker NJ. Mouse mammary tumour-like virus gene sequences and specific breast cancer morphology. J Clin Pathol (2007) 60:1071. doi:10.1136/jcp.2006.044487

46. Hoch-Marchaim H, Weiss AM, Bar-Sinai A, Fromer M, Adermann K, Hochman J. The leader peptide of MMTV env precursor localizes to the nucleoli in MMTV-derived T cell lymphomas and interacts with nucleolar protein B23. Virology (2003) 313:22-32. doi:10.1016/S0042-6822(03)00236-8

47. Vinner L, Mourier T, Friis-Nielsen J, Gniadecki R, Dybkaer K, Rosenberg $\mathrm{J}$, et al. Investigation of human cancers for retrovirus by low-stringency target enrichment and high-throughput sequencing. Sci Rep (2015) 5:13201. doi:10.1038/srep13201

48. Ford CE, Tran DD, Deng YM, Rawlinson WD, Lawson JS. Mouse mammary tumour like virus prevalence in breast tumours of Australian and Vietnamese women. Clin Cancer Res (2003) 9:1118-20.

49. Mazzanti CM, Al Hamad M, Fanelli G, Scatena C, Zammarchi F, Zavaglia K, et al. A mouse mammary tumor virus env-like exogenous sequence is strictly related to progression of human sporadic breast carcinoma. Am J Pathol (2011) 179:2083-90. doi:10.1016/j.ajpath.2011.06.046

50. Lawson JS, Ngan CC, Glenn WK, Tran DD. Mouse mammary tumour virus (MMTV) and human breast cancer with neuroendocrine differentiation. Infect Agent Cancer (2017) 12:24. doi:10.1186/s13027-017-0135-8

51. Pogo BG, Melana SM, Holland JF, Mandeli JF, Pilotti S, Casalini P, et al. Sequences homologous to the mouse mammary tumor virus env gene in human breast carcinoma correlate with overexpression of laminin receptor. Clin Cancer Res (1999) 5(8):2108-11.

Conflict of Interest Statement: No author has any financial, intellectual, or interpersonal conflicts of interest.

The reviewer $[\mathrm{AA}]$ declared a shared affiliation, with no collaboration, with one of the authors $[\mathrm{BY}]$ to the handling Editor.

Copyright $\odot 2018$ Lawson, Mazzanti, Civita, Menicagli, Ngan, Whitaker, Hochman, Braitbard, Yosufi and Glenn. This is an open-access article distributed under the terms of the Creative Commons Attribution License (CC BY). The use, distribution or reproduction in other forums is permitted, provided the original author $(s)$ and the copyright owner are credited and that the original publication in this journal is cited, in accordance with accepted academic practice. No use, distribution or reproduction is permitted which does not comply with these terms. 